\title{
Raman Spectroscopy: An Evaluation of Antimicrobial Characteristics of Technical Lignin
}

\author{
Klanarong Sriroth ${ }^{1 *}$ and Jackapon Sunthornvarabhas ${ }^{2}$ \\ ${ }^{1}$ MITR PHOL Group, Thailand \\ ${ }^{2}$ National Center for Genetic Engineering and Biotechnology, Thailand \\ *Corresponding author: Klanarong Sriroth, MITR PHOL Group, Phu Khiao, Thailand. \\ To Cite This Article: Klanarong Sriroth. Raman Spectroscopy: An Evaluation of Antimicrobial Characteristics of Technical Lignin. Am J Biomed \\ Sci \& Res. 2019 - 4(4). AJBSR.MS.ID.000809. DOI: 10.34297/AJBSR.2019.04.000809
}

Received: August 01, 2019 | Published: August 06, 2019

\begin{abstract}
Technical lignin from agricultural biomass has high potential for various applications and receives strong attention as one of the key materials in the future. One of its unique properties is antimicrobial property against wide range of microorganisms which is very helpful in medical and cosmetic application as potential natural antimicrobial agent. The current challenges are ability to swiftly identify its potency for both product development and routine analysis and its dark color from chromophores that were generated during delignification thus make it less attractive. Coniferyl alcohol, a chromophores and one of the three basic unit of lignin, is a key component that is responsible for antimicrobial property and also responsible for color generation of lignin after delignification. In order to control and monitor, it is essential to use characterization tools that is sensitive to the compound. Raman spectroscopy was reported to be the most efficient tools to monitor chromophores better than IR spectroscopic method. Therefore, using Raman spectroscopy to obtain tendency of antimicrobial property of lignin is suitable and appropriate.
\end{abstract}

Keywords: Technical lignin; Chromophores; Antimicrobial; Raman spectroscopy

\section{Technical Lignin from Agricultural By-Product}

Lignin, a large molecular weight polyphenol, receives significant attention during the last decade. Amount of technical reports per year were increased drastically from 2,6k documents in 2009 to $7.1 \mathrm{k}$ documents in 2019 based on a single keyword "lignin" [1]. Sharp increase suggests an attention on the subject. Several research groups and private companies heavily invest in research and development on the subject of biorefinery especially delignification and utilization of lignin with variation based on available feed stocks, technical expertise and intended product characteristics. Because difference in chemical characteristic of lignin was primary caused by type of feed stocks and method of delignification, chemical variation make lignin more versatile and attractive [2-5].

After a delignification, there are two available forms of lignin, dark color solid powder and solubilized lignin in solvent as dark color liquid, for utilization. Protolignin, lignin in native form, is almost colorless but gain color after delignification [6]. Technical lignin, lignin after delignification, has dark color was due to chromophores that were generated during delignification process [7]. It was reported that a more severe the extraction condition promotes chromophores and cause darker color in technical lignin [8]. Therefore, to obtain light color lignin, mild condition is essen tial. Figure 1 shows color of alkaline bagasse lignin at different alkaline concentration. Strong concentration promote darker colors. Delignification was performed by method described in [9] with variation in alkaline concentration.

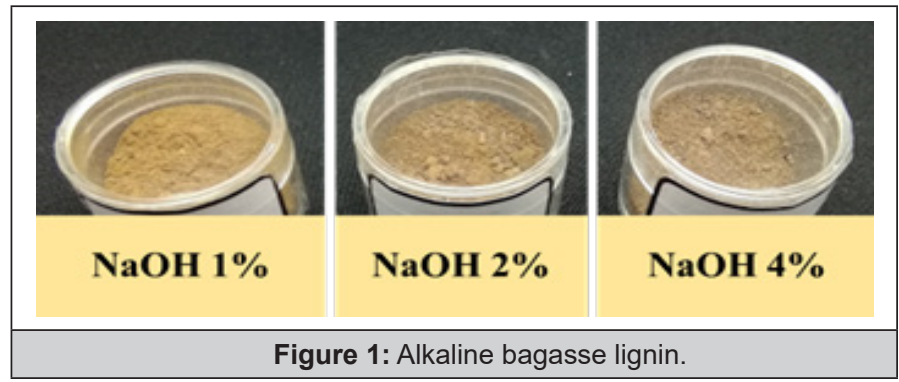

Utilization as Natural Antimicrobial Agents

Lignin is known for three major properties, antioxidant, anti-ultraviolet light and antimicrobial. Each property determines targeted application. Use of lignin as antioxidizing agent is suitable for medical application in which antioxidant property contribute and promote wound healing mechanism [10]. Use of lignin as anti-ultraviolet light is suitable for cosmetic product in which lignin act as UV light absorber [6]. Lignin as natural antimicrobial agent [11] receives less attention when compares to other properties. 
First report of lignin as potential antimicrobial agents was in 1979 [12]. However, small amount of publications discussed this aspect of lignin despite several decades of recognition of its property. This might due to its inferior performance when compare to inorganic antimicrobial agent, silver nanoparticles. In our previous study, it is possible to use alkaline bagasse lignin extracts as replacement of silver nanoparticle at controlled conditions [13]. The calculation was based on material price, antimicrobial performance, and effective concentration. It was stated that methoxyl group in two, coniferyl alcohol and sinapyl alcohol, of the three basic unit of lignin as shown in Figure 2 were responsible for antimicrobial characteristic [12]. Coniferyl alcohol is considered to be responsible for antimicrobial property in technical lignin and also recognized as color-generating compound despite small in quantities but difficult to separate [14]. This implies that correlation between antimicrobial property and degree of color of technical lignin are related as well as could be controlled and monitored through quantity of coniferyl alcohol.

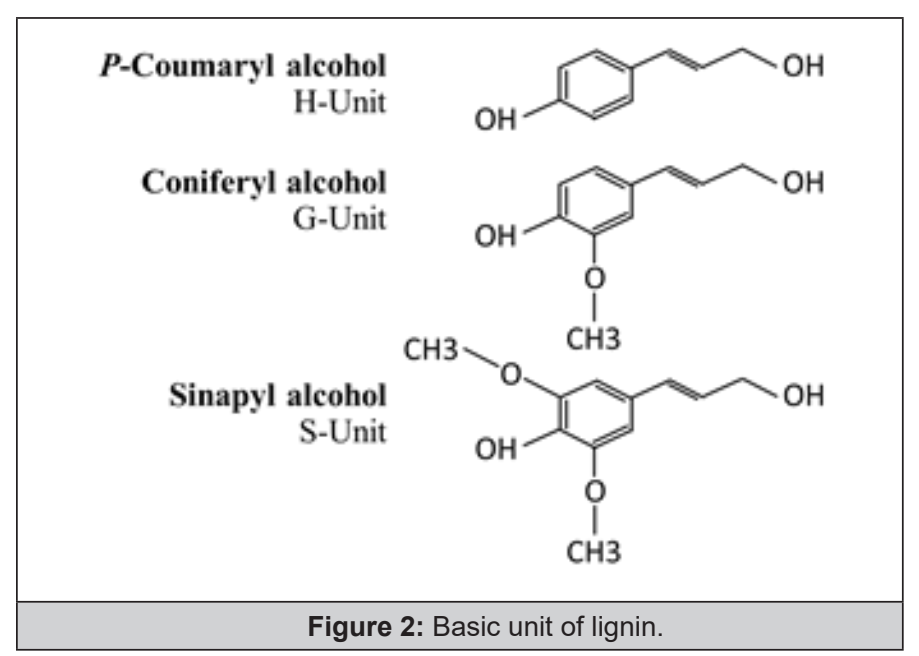

\section{Benefit of Raman Spectroscopy for Lignin Analysis}

An intensity of Raman spectroscopy is specific to molecular structure of compound. Although chromophores could be detected by IR spectroscopy, it requires high concentration unlike Raman spectroscopy. In addition to specificity of characterization method to targeted compound, Raman intensity could be quantified and related to amount of substance thus allow an accurate representation of compound within targeted and convert to specific property based on targeted compound [15]. By adopting this concept, as shown in Figure 3, to evaluate antimicrobial tendency of technical lignin from intensity of coniferyl alcohol found within sample, it is possible to swiftly identify antimicrobial tendency of targeted samples without conducting tedious susceptibility test of technical lignin with bacteria. However, this approach is not intended to be used as a replacement of susceptibility test since it can only reveal tendency and not actual concentration of technical lignin to inhibit (minimum inhibitory concentration, MIC) and kill (minimum bactericidal concentration, MBC) targeted bacteria. In addition, it is necessary to have a set of correlation between intensity of coniferyl alcohol at effective concentration against targeted microorganism as guideline for routine analysis. Therefore, this concept requires further investigation.

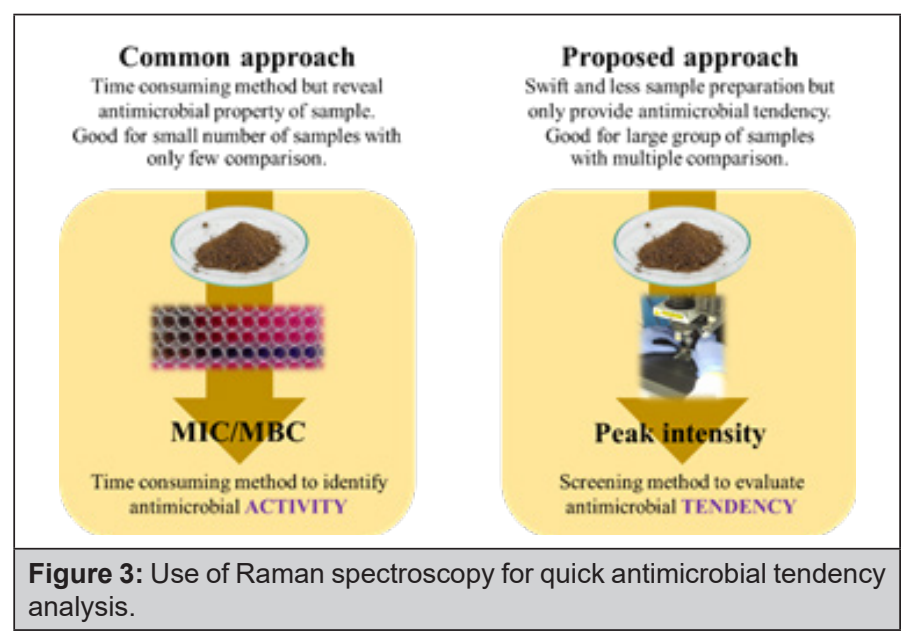

\section{Conclusion}

Antimicrobial characteristic of lignin from biomass and its color are related and controlled by basic unit structure. Due to strong sensitivity of Raman spectroscopy and ability to quantify amount of substance based on its intensity, it is possible quantify antimicrobial tendency of lignin from amount of coniferyl alcohol by Raman spectroscopic method. This approach is intended to be used as screening method. It should not be used as replacement to susceptibility test due to it cannot fully quantify antimicrobial property of substance.

\section{References}

1. www.sciencedirect.com, keyword: lignin, accessed July 24, 2019.

2. Rabemanolontsoa H, Sala S (2016) Various pretreatments of lignocellulosic. Bioresource Technology 199: 83-91.

3. Jönsson LJ, Martín C (2016) Pretreatment of lignocellulose: Formation of inhibitory by-products and strategies for minimizing their effects, Bioresour Technol 199: 103-112.

4. Chen H, Liu J, Chang X, Chen D, Xue Y, et al. (2017) A review on the pretreatment of lignocellulose for high-value chemicals, Fuel Processing Technology 160: 196-206.

5. Bhutto AW, Qureshi K, Harijan K, Abro R, Abbas T, et al. (2017) Insight into progress in pre-treatment of lignocellulosic biomass. Energy 122: 724-745.

6. Lee SC, Tran TMT, Choi JW, Won K (2019) Lignin for white natural sunscreens. Int J Biol Macromol 122: 549-554.

7. Qiu X, Yu J, Yang D, Wang J, Mo W, Qian Y (2018) Whitening sulfonated alkali lignin via $\mathrm{H} 202 / \mathrm{UV}$ radiation and its application as dye dispersant. ACS Sustainable Chemistry \& Engineering 6: 1055-1060.

8. Chen Y, Fan Y, Gao J, Li H (2012) Coloring characteristics of in situ lignin during heat treatment. Wood Science Technology 46: 33-40.

9. Sunthornvarabhas J, Liengprayoon S, Suwonsichon T (2017) Antimicrobial kinetic activities of lignin from sugarcane bagasse for textile product, Industrial Crops \& Products 109: 857-861.

10. Mahmood Z, Yameen M, Jahangeer M, Riaz M, Ghaffar A, et all. (2018) Lignin as Natural Antioxidant Capacity. 
11. Sriroth K, Sunthornvarabhas J (2018) Lignin from Sugar Process as Natural Antimicrobial Agent. Biochem Pharmacol (Los Angel) 7: 239.

12. Zemek J, Košíková B, Augustín J, Joniak D (1979) Antibiotic properties of lignin components. Folia Microbiol (Praha) 24(6): 483-486.

13. Sunthornvarabhas J, Liengprayoon S, Lerksamran T, Buratcharin C, Suwonsichon T, et al. (2019) Utilization of Lignin Extracts from Sugarcane Bagasse as Bio-based Antimicrobial Fabrics. Sugar Tech 21(2): 355-363.
14. Agarwal UP, Atalla RH (1999) Using Raman Soectroscopy to Identify Chromophores in Lignin-Lignocellulosics in Lignin: Historical, Biological, and materials Perspectives; ACS Symposiu Series; American Chemical Society, Washington, DC, USA.

15. Agarwal UP (2006) Raman imaging to investigate ultrastructure and composition of plant cell walls: distribution of lignin and cellulose in black spruce wood (Picea mariana), Planta 222: 1141-1153. 